\title{
Calibration of an EMG-Based Body Model with six Muscles to control a Leg Exoskeleton
}

\author{
Christian Fleischer and Günter Hommel
}

\begin{abstract}
This paper presents a body model of intermediate level of detail to allow prediction of the knee torque produced by thigh muscles based on EMG signals. This torque prediction is used as input for a torque controller that adapts the level of support offered to an operator by a powered leg orthosis. The level of detail of the body model is chosen in such a way, that all parameters of the model can be calibrated for a specific operator with only a few sensors that are mounted on the exoskeleton.
\end{abstract}

\section{INTRODUCTION}

Exoskeleton systems for human operators offer a variety of different applications ranging from support during a rehabilitation process, human power augmentation for factory workers or emergency personnel to force feedback for haptic interfaces in telemanipulation, games and entertainment. Depending on the size, weight, and handling of the devices, they could even be beneficial in everyday life for elderly oder disabled people.

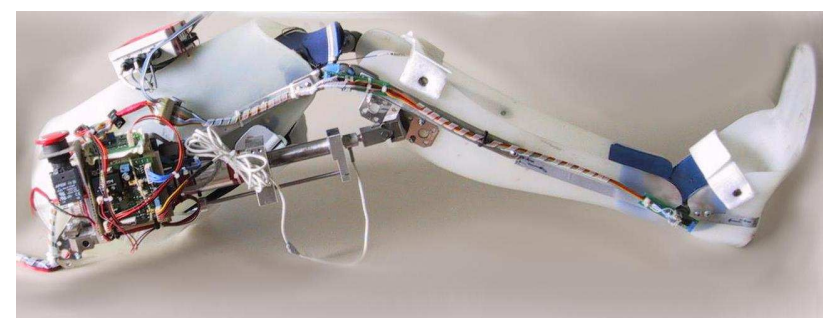

Fig. 1. Exoskeleton for the right leg.

Especially in recent years many research groups have shown interest in this topic: The Berkeley Lower Extremity Exoskeleton, for example, is a military exoskeleton to aid soldiers carrying heavy loads [1]. The Hybrid Assistive Leg is an actuated body suit for both legs [2], in the latest version extended for both arms. It is designed to support elderly people and as rehabilitation device. The powered lower limb orthosis described in [3] is developed to assist during motor rehabilitation after neurological injuries by relearning typical gait patterns.

Whatever the use of such an exoskeleton is, there is need of an interface between the device and the operator as soon as interaction is desired. One approach for an intuitive interface is to utilize electromyographic signals (EMG signals) emitted by muscles during their activation. Those signals can

C. Fleischer, G. Hommel are with the Institute of Computer Engineering and Microelectronics, Technische Universität Berlin, Sekretariat EN 10, Einsteinufer 17, D-10587 Berlin, Germany, \{fleischer, hommel\}@cs.tu-berlin.de be continually interpreted and used as input for a control structure that computes the appropriate support. The operator simply needs to try to perform the movement and will almost instantly get support from the exoskeleton.

When EMG signals are to be evaluated, the question of model complexity arises: In Lloyd [4] a promising but very complex musculoskeletal model is presented that takes into account 13 muscles crossing the knee to estimate the resulting knee torque offline. In [5] EMG signals of the biceps brachii and triceps brachii where used to estimate the elbow joint moment. A moment controller was fed to control a two-link exoskeletal arm to lift an external load with the hand. In [6] an EMG-based control scheme for HAL is described with a very simple body model.

The problem of complexity is, that the more muscles are incorporated into the model, the more parameters have to be determined. All EMG-related parameters are unfortunately subject dependent and even change from day to day due to varying conditions of the skin, blood circulation etc. [7]. In our case, to allow easy application of the exoskeleton, the calibration should be performed with sensors mounted on the orthosis alone with a limited and short set of calibration movements. This results in a limited set of reference values to optimize the parameters with, making it impossible to identify many parameters. On the other hand, if not all relevant muscles are taken into account, the exoskeleton support that is computed based on the EMG signals might not be sufficient in all phases of interesting movements.

The exoskeleton for which the control structure is implemented covers the right leg and actuates the knee joint with an electrical linear actuator as shown in fig. 1 [8]. This work substantially improves previous work by us [9], [10].

\section{CONTROL STRUCTURE}

The algorithm estimates the current muscle forces from the EMG signals and computes the resulting knee torque through a simplified body model as described later. The desired support torque is a function of the operator's own torque contribution and a given support ratio. This support ratio can be adjusted to the needs of the operator but is not changed during a particular experiment. The current knee torque is calculated by the measurement from the force sensor at the tip of the actuator, taking into account the geometry of the actuator and the current knee angle. The difference of the desired supporting torque (target knee torque) and the current knee torque forms the torque error that is passed to the torque controller. The controller sets the control signals for the actuation accordingly (refer to fig. 2). 
Since the exoskeleton can only offer support for the knee joint, it is feasible to read the EMG signals from muscles spanning the knee. Depending on the kind of movement that is performed, different muscles are activated and in varying order. Unfortunately it is not possible to measure all muscles with surface electrodes: Some muscles are located too deep close to the thigh bone.

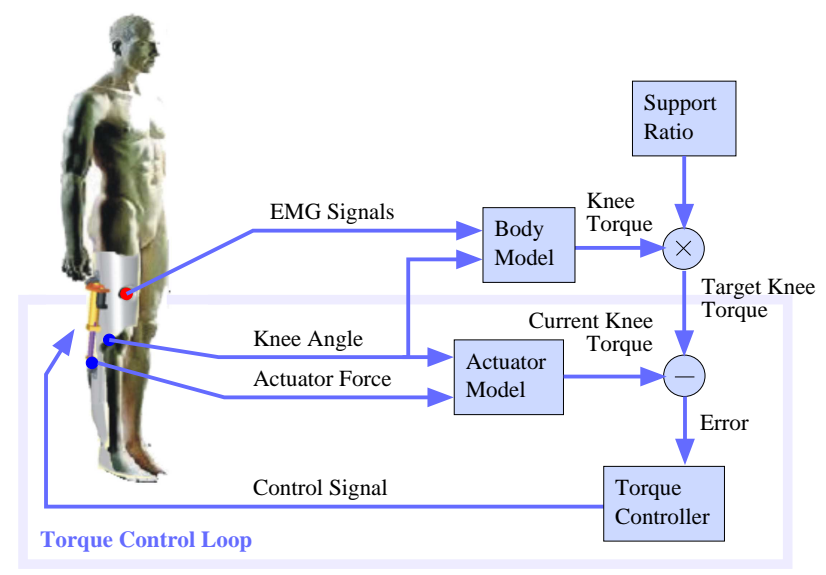

Fig. 2. Control structure of the system.

It is also reasonable to select muscles according to their proximity to the skin and their physiological cross-sectional area (PCA, approximately linear related to maximum force [11]) to get a good estimation of the overall force production.

The muscles selected for this work with their relative PCA are: (1) the rectus femoris (8\%), (2) vastus medialis (15\%), (3) vastus lateralis $(20 \%)$, (4) the semimembranosus $(10 \%)$, (5) semitendinosus (3\%), and (6) biceps femoris (10\%), covering a total of $66 \%$ of the cross-sectional area of all thigh muscles [12]. Electrodes have been placed according to recommendations in [13].

The raw EMG signal is postprocessed with offset elimination, rectification and application of a second order Butterworth lowpass filter with a cutoff frequency of $1.6 \mathrm{~Hz}$, forming the muscle activation $u(t)$. To compute the resulting joint torque based on the EMG signals, several steps have to be performed which are summarized here. They are similar to models presented in [14], [15].

The postprocessed EMG signal, $u(t)$, is converted into a muscle activation (based on [16][4]) by

$$
a(u)=\frac{e^{A u R^{-1}}-1}{e^{A}-1}
$$

where $R$ is the estimated maximum EMG value, and $A$ a non-linear shape factor. The muscle force is produced by an active and passive component, taking into account the activation, $a(u)$,

$$
F^{m}=\left(f_{A}\left(\tilde{l}^{m}\right) a(u)+f_{P}\left(\tilde{l}^{m}\right)\right) F_{o}^{m}
$$

with the active force-length curve, $f_{A}\left(\tilde{l}^{m}\right)$, and the passive force-length curve, $f_{P}\left(\tilde{l}^{m}\right)$ as functions of the normalized length of the muscle fibers, $\tilde{l}^{m}$. Those curves take into account that force output of muscle fibers depends on their length and can be found in literature [15]. One way to obtain the muscle fiber length is to compute the length of the muscle and tendon together (musculotendon unit) with a human skeleton model, as presented, for example, in [15]. This delivers the length of the musculotendon unit, $l^{m t}$. We assume that the tendon is stiff and length change is performed by the muscle fibers, so that the normalized length of the muscle fibers, $\tilde{l}^{m}$, can be computed by

$$
\tilde{l}^{m}=\frac{l^{m t}-s \hat{l}_{s}^{t}}{s \hat{l}_{s}^{t}}
$$

where $\tilde{l}_{s}^{t}$ is the tendon slack length according to literature, and $s$ a parameter to fit the value from literature to the operator. The pennation angle of muscles is neglected here.

Performing the above mentioned computation for every muscle $i$, with $0 \leq i<N$, and taking into account their respective moment arms, $r_{i}$, from the skeleton model yields for the resulting torque:

$$
T=\sum_{i=0}^{N-1} r_{i} F_{i}^{m}
$$

Due to all simplifications of this model, $T$ is only a rough estimation of the torque produced by the muscles of the operator.

The EMG-related parameters of this model are the maximum expected EMG signal, $R$, the non-linear shape factor of the activation function, $A$, and the maximum muscle force, $F_{o}^{m}$. The geometry-related parameter is the scale of the tendon slack length, $s$. Those four parameters have to be calibrated for every muscle. The geometry parameters are only required to be calibrated once for every operator, whereas the EMG-related parameters have to re-calibrated for every experimental session.

The supporting torque of the exoskeleton is computed with a linear relationship by

$$
T_{s}=G \cdot T
$$

where $G$ is the gain of the amplification, and $T$ the torque from eq. 4. $T_{s}$ is the target value for the torque controller that generates appropriate signals so that the actuation of the exoskeleton contributes the desired amount of torque to the movement (refer to fig. 2). The current knee torque from the actuator, $T_{A}$, is obtained through the force sensor that is attached in-series with the linear actuator, as shown in fig. 1.

\section{PARAMETER CALIBRATION}

There are four parameters $\left(A_{i}, R_{i}, F_{o, i}^{m}, s_{i}\right)$ for every one of the six muscles that have to be calibrated.

During the description of the calibration algorithm some assumptions are made to simplify the process. Furthermore it has to be kept in mind, that the calibration is used to control an exoskeleton system. The accuracy of the calibration is not the most important aspect. It is rather desirable that the exoskeleton behaves in a predictable manner so that the 
operator feels comfortable and can take advantage of the offered support.

Unfortunately, direct measurement of reference values for the calibration is limited to the force sensor attached in-series with the actuator. If the actuator is locked (allowing no length change) this sensor measures the resultant of all forces acting on the thigh and shank (contact and gravitational forces), and the forces of muscles spanning the knee.

The calibration setup has to be chosen in such a way that effects of external forces can be taken into account without measuring them directly. The selected movements have to activate all muscles that need to be calibrated in a wide range to allow a feasible calibration. Unfortunately this is not possible by only using the force sensor of the exoskeleton. But muscle forces can also be estimated indirectly by measuring the resulting movement. With a simplified model of the human body the muscle forces that must have existed to perform the recorded movement can be estimated.

\section{A. Calibration Procedure}

The calibration procedure is composed of two steps: During the first step the operator is sitting on a chair with the exoskeleton shank not having any contact with the environment, the thigh supported by the chair, and the knee comfortably flexed. The actuator is locked, allowing only isometric contractions. When the thigh muscles are relaxed, the force measured by the force sensor is a result of gravitation acting on the exoskeleton and the embraced leg leading to a torque offset, $T_{G}$. After that, the operator tries to extend and flex the knee with maximum muscle activation in both directions a few times. The measured force is now an overlay of all active muscles and the influence of gravitation. This is performed under several different joint angles to get reference values with different muscle fiber lengths for the geometry calibration.

During the first step the vastus medialis and the vastus lateralis are not or only a little active. They get mainly activated when a large knee torque is required as during standing up from a chair and climbing stairs or when the leg is almost extended. Since those movements should be supported by the exoskeleton, those muscle activations also have to be evaluated, requiring the second step of the calibration procedure.

During the second step, the operator is initially sitting on a chair with both legs parallel and both feet on the ground. The actuator is detached, allowing free motion in the knee joint. The operator is slowly getting up from the chair, not supporting himself or herself with his or her arms and in complete balance without moving the feet. The movement of the operator is evaluated by a dynamic body model. By computing the inverse dynamics it is possible to estimate the torque in the knee that the muscle must have produced to create the recorded movement. The strict limitations to the movement are very important to allow necessary simplifications of the model and eliminate the need for many additional sensors.

\section{B. Calibration Algorithm}

The calibration process is subdivided into several steps: The first step collects data while the muscles are activated. When sufficient data have been stored, the parameter calibration is executed in step two.

1) Data Collection: During calibration the postprocessed signal $u_{i}(t)$ of EMG sensor $i$, the knee angle $q_{2}(t)$, the ankle angle $q_{1}(t)$, and the force sensor value $F_{A}(t)$ are recorded. The delay of the EMG postprocessing is compensated by a delay buffer for all other signals. Thus, $u_{i}, q_{1}, q_{2}$, and $F_{A}$ denote digitized sensor readings from the same point in time.

The data collection routine ensures that samples with different levels of muscle activationa are recorded, with the amount of data kept in reasonable limits.

In our algorithm, the data is stored in tables, one table for every muscle and every trial. The table index, $h_{i, k}$ for muscle $i$ and trial $k$ is computed by the EMG value, $u_{i}$ of muscle $i$, and the entry-width, $S_{i}$, by

$$
h_{i, k}=\left\lfloor u_{i} S_{i}\right\rfloor
$$

The values stored in every entry are: $u_{i}, q_{1}, q_{2}$, the reference torque in the knee joint, $T_{R}$, and the number of updates of the same entry, $n_{u}$. The new values are averaged on a per-element basis with previous values of the same entry weighted with $n_{u}$. Depending on the calibration step, the reference torque, $T_{R}$, is derived from measurements of the force sensor, or computed by inverse dynamics:

$$
T_{R}= \begin{cases}-\left(T_{A}-T_{G}\right) & \text { during step 1 } \\ T_{D} & \text { during step 2 }\end{cases}
$$

The computation of $T_{D}$ (knee torque calculated by inverse dynamics) is described later in this section. For step two the data collection is started after the knee angle has changed by $10^{\circ}$ indicating that the standup movement has begun and the chair is not longer touched.

All this ensures that the stored data contains samples from different levels of activation of the muscles without collecting too much data. Optimization has started after all required movements have been performed.

2) Force Determination of Individual Muscles: The calibration process described below is not a global optimization for all parameters at once over all data. Some muscles are cooperating during the described exercises, making it impossible for the optimization algorithm to distinguish between the individual muscle contributions. The reason for not merging all muscles into a single muscle is, that during different non-isometric tasks, the muscles have different cooperation patterns.

To compute the individual muscle forces, $F_{i}^{m}$, the reference torque, $T_{R}$, has to be split up among all active muscles. The individual torque contribution of a muscle, $T_{i}$, 
is computed from the reference torque through:

$$
T_{i}=T_{R} \frac{A_{i}}{\sum A_{i}}
$$

with

$$
A_{i}= \begin{cases}P C A_{i} \cdot a_{i}\left(u_{i}\right) & \text { if already calibrated } \\ P C A_{i} \cdot \frac{u_{i}}{R_{i}} & \text { otherwise }\end{cases}
$$

where $R_{i}$ is the maximum recorded EMG signal of muscle $i$ and $u_{i}$ the postprocessed EMG signal. The required activation, $a_{i}\left(u_{i}\right)$ is only available if this calibration has been performed before, and parameters from the previous run are available. Otherwise the activation function is approximated by a linear relationship. Dividing $T_{i}$ by the moment arm, $r_{i}$, yields the individual muscle force, $F_{i}^{m}$. We now have a relationship between different muscle activations (through equations 6) and associated muscle forces for every entry of every table of a particular muscle.

3) Parameter Optimization: First the geometry parameters of all muscles are calibrated. The parameters $s_{i}$ have to be determined in such a way that the EMG-to-force relationship is consistent for all joint angles. For a particular muscle this can be achieved by minimizing the standard deviation of the individual muscle forces, through optimization of the tendon slack length scale, $s_{i}$. Since $s_{i}$ can be bound to an interval of $[0.85,1.25]$ (experimentally determined) this can be performed with a linear minimum search and a fixed stepsize. The effect of optimizing the geometry is shown in fig. 3 .

The EMG-parameter optimization is a curve fitting problem of the EMG-to-force function with data points taken from all tables of the muscle that is to be optimized. The range of the postprocessed EMG signal, $R_{i}$, can be immediately taken from the highest entry of all tables of the muscle. In theory, $F_{o}^{m}$ could be set to the individual muscle force of the same entry. But due to measurement inaccuracies it is recommended to calibrate it.

The shape $A_{i}$ of the function can be bound to an interval of $[-5,0[$. This is reasonable when looking at the resulting functions: The function is approximating a linear relationship for $A_{i} \rightarrow 0$. For $A_{i} \rightarrow-5$ the curvature increases. If too large, changes of the EMG signals in the upper part of the range do not result in significant changes of muscle force. This indicates that some error during data collection occured.

The calibration can be performed with any optimization algorithm. The total error of a calibration step is given as a function of the shape and force parameters,

$$
E\left(A, F_{o}^{m}\right)=\sum_{k} \sum_{h}\left(a\left(u_{k, h}\right) F_{o}^{m}-F_{k, h}^{m}\right)^{2}
$$

where $F_{k, h}^{m}$ is the individual muscle force computed for entry $h$ of table $k$ of the muscle to be optimized, and $u_{k, h}$ is the EMG value of the same entry.

4) Torque Estimation with a Dynamic Human Body Model: To estimate the knee torque the muscles are producing during getting up from a chair without hindering the movement, it is necessary to track the movement and compute the torque by inverse dynamics. The advantage of using a rather simple movement, as described in sec. III-A, is the possibility to apply major simplifications. Those are:

- The model is 2-dimensional.

- Both legs are merged into one.

- The operator is not allowed to support himself with e.g. his arms to omit unmeasured external force input.

- Only trunk, thigh, and shank are modelled. Arms and head are integrated in the trunk properties.

- The ankle is rigidly attached to the floor.

- Joint friction and passive joint stiffness is neglected (typically $2-5 \mathrm{Nm} / \mathrm{rad}$ in the mid-range of motion[17]).

- Joint accelerations are small and can be neglected.

- Joint velocities (typically below $100^{\circ} / \mathrm{s}$ ) during the considered movements contribute about $4 \%$ of the knee joint torque and are neglected.

Following the model description above, the parameters of the model are:

- The total body mass of the human: $m_{\text {total }}$.

- Masses for trunk, thigh and shank (taking into account the merging of limbs) as a fraction of $m_{\text {total }}$ are $m_{u}=$ $0.628, m_{t}=0.2, m_{s}=0.93$ [12].

- Length and width of the trunk, thigh and shank: $\left(L_{u}, W_{u}\right),\left(L_{t}, W_{t}\right),\left(L_{s}, W_{s}\right)$.

- The location of the center of mass as a fraction of the length of the body segment from the proximal end for each body segment. For the thigh, and shank they are $r_{t}=0.433$ and $r_{s}=0.433$ [12]. The mass center of the trunk $r_{u}$ is determined separately later on.

The model includes three joints: ankle, knee, hip. The third angle can be computed as a result of the other two angles, by introducing an additional constraint: The center of mass $(\mathrm{CoM})$ of the body projected onto the ground must be on a specific point within the region of the foot: the balance point $\vec{B}=\left(b_{x}, 0\right)^{T}$ with $b_{x}=0.04 \mathrm{~m}$. Since the movement is slow a criterion for static balance can be used.

The angle of the hip can be calculated by:

$$
q_{3}= \begin{cases}-q_{1}-q_{2} & \text { if } C>+1 \\ \pi-q_{1}-q_{2} & \text { if } C<-1 \\ \arccos (C)-q_{1}-q_{2} & \text { otherwise }\end{cases}
$$

with

$$
\begin{aligned}
C= & \frac{A}{B} \\
A= & b_{x} m_{\text {total }}-L_{s}\left(m_{\text {total }}\left(1+r_{s}\right)\right) \cos \left(q_{1}\right) \\
& -L_{t}\left(m_{u}+m_{t}\left(1+r_{t}\right)\right) \cos \left(q_{1}+q_{2}\right) \\
B= & L_{u} r_{u} m_{u} \\
m_{\text {total }}= & m_{s}+m_{t}+m_{u}
\end{aligned}
$$

where $q_{1}, q_{2}, q_{3}$ are the ankle, knee, and hip angles respectively. If the argument $C>+1$ or $C<-1$ the balancing condition is violated: $C o M_{x} \neq b_{x}$ and $\arccos (C)$ cannot be computed. To allow calculation of an approximated hip angle, $C$ is replaced by the boundary it has exceeded.

Keeping the balance point $\vec{B}$ fixed over the whole movement is a rough approximation: In normal movement this 


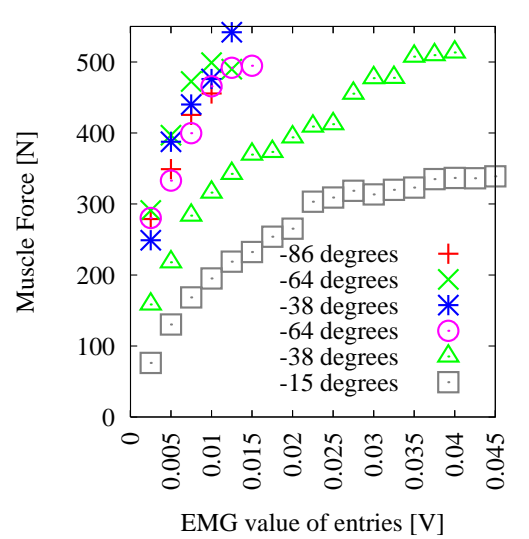

Data stored in tables
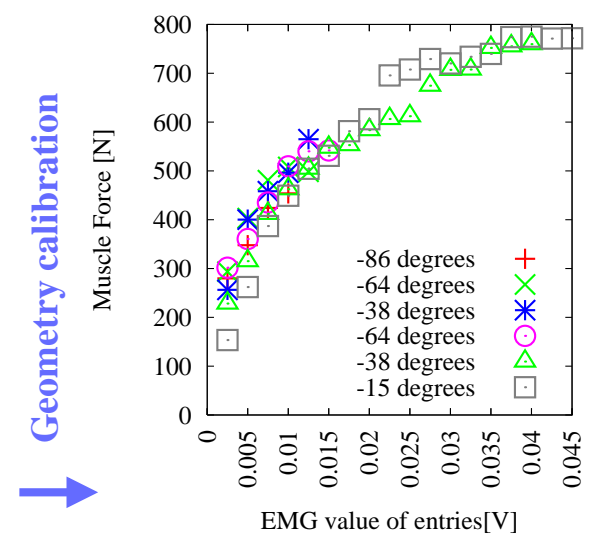

Data with regard to geometry

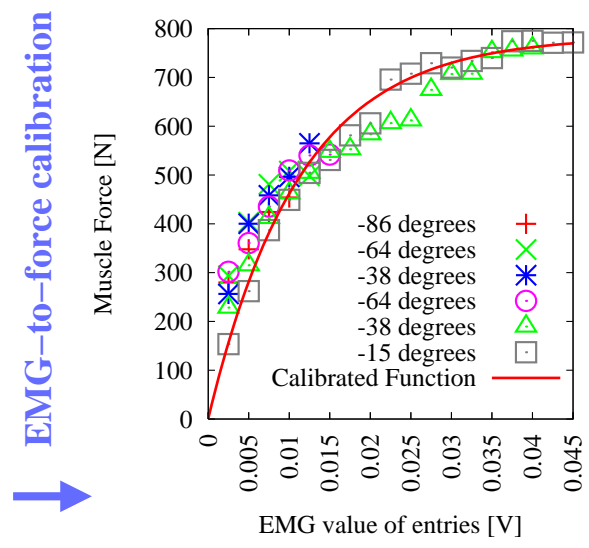

Interpolated EMG-to-force function

Fig. 3. Effect of the calibration for one specific muscle: Left: The individual muscle force plotted against the EMG values of all tables. Center: Estimated muscle forces with geometry model. Right: Interpolated EMG-to-force function.

point moves and is counterbalanced by motions in the joints to maintain a stable pose.

For this simplified body model we only need one additional sensor for measuring the ankle angle $q_{1}$. The formula for the knee torque $T_{k}$ with the simplifications justified above (joint accelerations and velocities are 0: $\dot{u}_{1}=\dot{u}_{2}=\dot{u}_{3}=$ $u_{1}=u_{2}=u_{3}=0$ ) is:

$$
\begin{aligned}
T_{k}= & -g\left(L_{t} m_{t}\left(1+r_{t}\right) \cos \left(q_{1}+q_{2}\right)\right. \\
& +m_{u}\left(L_{t} \cos \left(q_{1}+q_{2}\right)\right. \\
& \left.\left.+L_{u} r_{u} \cos \left(q_{1}+q_{2}+q_{3}\right)\right)\right)
\end{aligned}
$$

The dynamical equations have been computed with the tool AutoLev [18]. The script for the model definition can be received from the authors on request.

Assuming that $-1 \leq C \leq+1$ and substituting $q_{3}$ in eq. 12 with the expression from eq. 11 yields:

$$
\begin{aligned}
T_{k}= & -g\left(L_{t} m_{t}\left(1+r_{t}\right) \cos \left(q_{1}+q_{2}\right)\right. \\
& +m_{u} L_{t} \cos \left(q_{1}+q_{2}\right)+b_{x} m_{\text {total }} \\
& -L_{s}\left(m_{\text {total }}\left(1+r_{s}\right)\right) \cos \left(q_{1}\right) \\
& \left.-L_{t}\left(m_{u}+m_{t}\left(1+r_{t}\right)\right) \cos \left(q_{1}+q_{2}\right)\right)
\end{aligned}
$$

The knee torque is not depending on $r_{u}$ or $q_{3}$. The mass center of the trunk $r_{u}$ should be chosen in such a way that the balance condition is fulfilled throughout the standupmovement. Evaluating eq. 11 for $r_{u}$ at one important extreme of the movement (initial phase when losing contact with the chair) yields

$$
\begin{aligned}
r_{u}= & -\left[L_{s}\left(m_{t}+m_{u}+m_{s}\left(1+r_{s}\right)\right) \cos \left(q_{1}\right)\right. \\
& +L_{t}\left(m_{u}+m_{t}\left(1+r_{t}\right)\right) \cos \left(q_{1}+q_{2}\right) \\
& \left.-b_{x}\left(m_{s}+m_{t}+m_{u}\right)\right] \\
& {\left[L_{u} m_{u} \cos \left(q_{1}+q_{2}+q_{3}\right)\right]^{-1}+r_{\Delta} }
\end{aligned}
$$

The contribution $r_{\Delta} \approx 0.2$ moves the mass center a little towards the distal end (to the head) to be on the safe side for repeated measurements: $C$ only exceeds the upper boundary
+1 when the CoM cannot be brought over the balance point due to the knee and ankle configuration.

Unfortunately, the $x$-coordinate of the balance point $\vec{B}$ appears linearly in eq. 13 multiplied by the total body mass $m_{\text {total }}$. As a consequence, variation of the balance point has significant influence on the computed knee torque.

5) Repeated Optimizations: The distribution of the reference torque to the individual muscle in eq. 8 can take into account results from previous optimizations. And computing the passive force to calculate the geometry error requires a tendon slack length scale from a previous run or has to be omitted. Experiments have shown that those aspects influence the results only slightly, and an optimization should only be repeated once or twice (both, geometry and EMG calibration). More iterations do not significantly improve the results, and slow drifting of the results can occur due to coactivation of the muscles. In that case the optimization behaves like a global optimization that tries to optimize all parameters at once and cannot distinguish between the individual contributions properly.

\section{EXPERIMENTS AND RESULTS}

A re-run of data with the calibrated parameters of the isometric flexion and extension task is shown in fig. 4. It can be seen that the calibration is consistent in itself, so that the sum of muscle torque contributions is almost equal to the reference torque, especially for the isometric flexion. Figure 5 shows a replay of the standup experiment. At $t \approx 1.6 \mathrm{~s}$ the contact with the chair was lost and the data collection was started. At $t \approx 4.5 \mathrm{~s}$ the subject was standing upright. It can be seen that for $t>4.2 \mathrm{~s}$ the predicted torque is smaller than the torque based on the inverse dynamics. Due to the balancing condition the thigh and trunk is not upright, but the knee and hip are slightly flexed. This results in a residual torque which is not present in the human. This could be omitted, if the balance point would be allowed to move on a trajectory, and if this trajectory was known. Unfortunately it is not, which decreases the predictability of the model: 

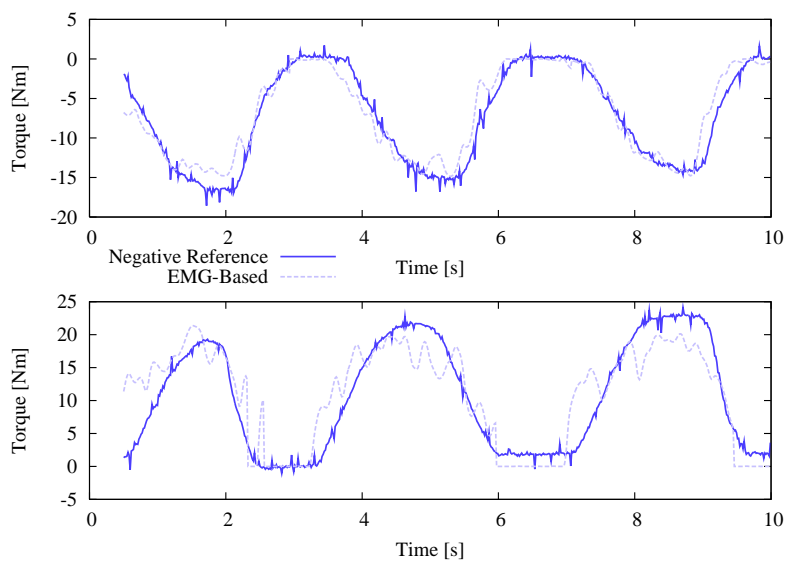

Fig. 4. Re-run of the isometric flexion (top) and extension (bottom) data with the calibrated EMG-to-force functions.
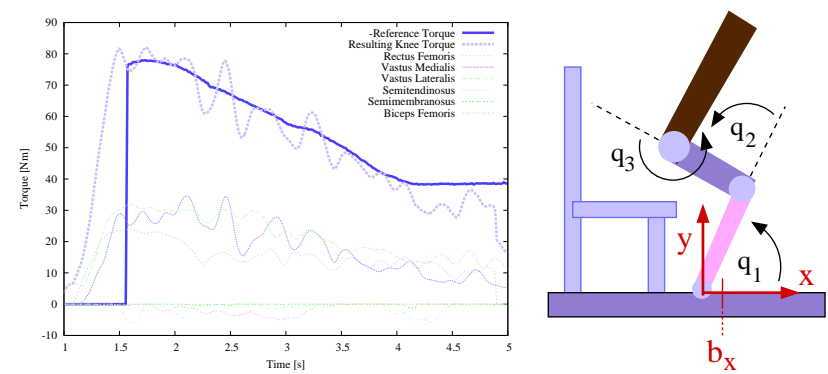

Fig. 5. Left: data from the standup experiment after calibration. Right: angles of the body model.

Since all standup movments are performed slightly different, the true balance point is deviating from the point of the model leading to an error in the estimation. This can be observed, if the calibrated parameters are applied to data from a different trial. While the isometric predictions show similar good results, the torques computed from the inverse dynamics and derived from the EMG signal show a considerable error of $20 \%$ or more. By adjusting the balance point the reference data can be manipulated to fit the predicted data.

Smoother curves of the EMG signal can hardly be reached by a more accurate calibration. It might be minimized by a more detailed activation model or by filtering the signal with a lower cutoff frequency. But when using the powered exoskeleton this would result in a slow response of the system due to the delay in the lowpass filter.

\section{CONCLUSION}

In this paper an algorithm has been proposed that can calibrate a simplified EMG-to-force relationship for six muscles with a minimum of sensors that can all be mounted on an exoskeleton. The relationship is optimized based on isometric force sensor measurements and evaluation of a dynamic body model during the standup task. Coactivation and cocontraction of muscles is considered by the algorithm.
Properties of the muscles that are mainly important during faster movements (running, cycling etc.), like the forcevelocity relationship, are not considered currently.

Unfortunately the algorithm is very sensitive to the position of the balance point. Further experiments have to be performed to investigate if a strategy for the proper estimation of the position of the balance point based on human behavior can be found. An alternative could be to model the trajectory of the balance point as a function with parameters which are also optimized, but without overfitting the model to a specific data set.

\section{REFERENCES}

[1] H. Kazerooni and R. Steger, "The Berkeley Lower Extremity Exoskeleton," Journal of Dynamic Systems, Measurement, and Control, vol. 128, pp. 14-25, 2006.

[2] H. Kawamoto and Y. Sankai, "Power assist method based on phase sequence driven by interaction between human and robot suit," 13th IEEE International Workshop on Robot and Human Interactive Communication, 2004, pp. 491-496, 2004.

[3] G. Sawicki, K. Gordon, and D. Ferris, "Powered Lower Limb Orthoses: Applications in Motor Adaptation and Rehabilitation," Rehabilitation Robotics, 2005. ICORR 2005. 9th International Conference on, pp. 206-211, 1928.

[4] D. G. Lloyd and T. F. Besier, "An EMG-driven musculoskeletal model to estimate muscle forces and knee joint moments in vivo," Journal of Biomechanics, vol. 36, pp. 765-776, 2003.

[5] J. Rosen, M. Brand, M. B. Fuchs, and M. Arcan, "A myosignal-based powered exoskeleton system," in IEEE Transactions on Systems, Man, and Cybernetics, vol. 31, 2001.

[6] H. Kawamoto and Y. Sankai, "Comfortable power assist control method for walking aid by HAL-3," Systems, Man and Cybernetics, 2002 IEEE International Conference on, vol. 4, p. 6, 2002.

[7] J. V. Basmajian and C. J. De Luca, Muscles Alive: Their Functions Revealed by Electromyography. Williams \& Wilkins, 1985.

[8] C. Fleischer and G. Hommel, "Torque control of an exoskeletal knee with emg signals," in Proceedings of the Joint Conf. on Robotics: ISR 2006 and Robotik 2006, 2006.

[9] C. Fleischer, K. Kondak, C. Reinicke, and G. Hommel, "Online calibration of the emg-to-force relationship," in Proceedings of the IEEE/RSJ Int. Conf. on Intelligent Robots and Systems, 2004.

[10] C. Fleischer and G. Hommel, "Predicting the intended motion with EMG signals for an exoskeleton orthosis controller," in Proceedings of the IEEE/RSJ Int. Conf. on Intelligent Robots and Systems, 2005.

[11] R. Maughan, J. Watson, and J. Weir, "Strength and cross-sectional area of human skeletal muscle," The Journal of Physiology, vol. 338, no. 1 , pp. 37-49, 1983.

[12] D. A. Winter, Biomechanics and Motor Control of Human Movement. John Wiley \& Sons, Inc., 1990.

[13] H. Hermens, B. Freriks, R. Merletti, D. Stegeman, J. Blok, G. Rau, C. Disselhorst-Klug, and G. Hägg, European recommendations for surface electromyography: results of the SENIAM project. Roessingh Research and Development.

[14] T. Buchanan, D. Lloyd, K. Manal, and T. Besier, "Neuromusculoskeletal Modeling: Estimation of Muscle Forces and Joint Moments and Movements From Measurements of Neural Command," J. Appl. Biomech, vol. 20, pp. 367-395, 2004.

[15] S. Delp, J. Loan, M. Hoy, F. Zajac, E. Topp, and J. Rosen, "An interactive graphics-based model of the lower extremity to studyorthopaedic surgical procedures," Biomedical Engineering, IEEE Transactions on, vol. 37, no. 8, pp. 757-767, 1990.

[16] J. Potvin, R. Norman, and S. McGill, "Mechanically corrected EMG for the continuous estimation of erector spinae muscle loading during repetitive lifting," European Journal of Applied Physiology and Occupational Physiology, vol. 74, pp. 119-132, 1996.

[17] F. Zajac and J. Winters, "Modeling Musculoskeletal Movement Systems: Joint and Body Segmental Dynamics, Musculoskeletal Actuation, and Neuromuscular control," Multiple Muscle systems, JM Winters and SL-Y Woo editors. Springer Verlag, New York, 1990.

[18] Kane Dynamics, Inc., http://www.autolev.com. 